\title{
Perioperative management of a patient with von Recklinghausen's disease who was expected to have difficulty in airway management:
}

\section{A case report}

\author{
Aiji Sato $(\text { Boku })^{1}$, Izumi Kuroda ${ }^{1}$, Hideto Imura ${ }^{1}$, Mayumi Hashimoto ${ }^{1}$, Yoko Okumura ${ }^{1}$, \\ Naoko Tachi ${ }^{1}$, Nagato Natsume ${ }^{1}$, and Masahiro Okuda ${ }^{1}$ \\ ${ }^{1}$ Aichi Gakuin University School of Dentistry
}

January 27, 2022

\begin{abstract}
Von Recklinghausen's disease is characterized by skin pigmentation, multiple neurofibromatosis, and osseous changes. In the management of anesthesia, a variety of pathologies need to be taken into account. This case describes the perioperative management of a patient with Recklinghausen's disease suspected difficulty in airway management.
\end{abstract}

Title: Perioperative management of a patient with von Recklinghausen's disease who was expected to have difficulty in airway management: A case report

Authors: Aiji Sato (Boku) D.D.S., Ph.D. ${ }^{1}$, Izumi Kuroda D.D.S. ${ }^{1}$, Hideto Imura D.D.S., Ph.D. ${ }^{2}$, Mayumi Hashimoto D.D.S. ${ }^{1}$, Naoko Tachi D.D.S., Ph.D. ${ }^{1}$, Yoko Okumura D.D.S. ${ }^{1}$, Nagato Natsume D.D.S., Ph.D. ${ }^{2}$, Masahiro Okuda M.D., Ph.D. ${ }^{1}$

Institutional affiliations and address for each author

${ }^{1}$ Department of Anesthesiology, Aichi Gakuin University School of Dentistry

${ }^{2}$ Cleft lip and palate center, Aichi Gakuin University Dental Hospital

\section{Corresponding author}

Aiji Sato (Boku), Department of Anesthesiology, Aichi Gakuin University School of Dentistry 2-11 Suemoridori, Chikusaku, Nagoya 464-8651, Japan

Tel: +81-52-759-2102, Fax; +81-52-759-2102, Email: bokuaiji@dpc.aichi-gakuin.ac.jp

\section{Key Clinical Message}

In the anesthetic management of patients with von Willebrand's disease, it is important to provide appropriate airway management, taking into account the cutaneous laxity caused by neurofibromatosis of Recklinghausen's disease.

\begin{abstract}
Von Recklinghausen's disease is characterized by skin pigmentation, multiple neurofibromatosis, and osseous changes. In the management of anesthesia, a variety of pathologies need to be taken into account. This case describes the perioperative management of a patient with Recklinghausen's disease suspected difficulty in airway management.
\end{abstract}

Keywords: Von Recklinghausen's disease, Awake intubation, Airway management 


\section{Introduction}

Von Recklinghausen's disease, which was first reported by Friedrich Daniel von Recklinghausen in 1882, is characterized by skin pigmentation, multiple neurofibromatosis, and osseous changes. ${ }^{1}$ The disease occurs in 1 out of 3000-4000 individuals. ${ }^{2}$ Problems in anesthesia management include hypertension caused by renal artery stenosis and circulation instability and major bleeding caused by pheochromocytoma ${ }^{3-5}$; airway obstruction caused by a tumor in the mouth, pharynx, or airways ${ }^{6}$; and an aberrant response to muscle relaxants. ${ }^{7,8}$ Here, we report the perioperative management of a patient with suspected difficulty in airway management because of cutaneous laxity caused by neurofibromatosis of Recklinghausen's disease and trismus resulting from pathological fractures associated with mandibular osteomyelitis. The patient provided written informed consent.

\section{Case Report}

A 74-year-old woman (height, $151 \mathrm{~cm}$; weight, $58 \mathrm{~kg}$ ) was scheduled for tooth extraction and antiinflammatory surgery under general anesthesia for right mandibular osteomyelitis. She had been diagnosed with von Recklinghausen's disease; her mother also had the same disease. Her past medical history included surgery for appendicitis when she was 50 years of age. Her preoperative examination findings, including blood tests, chest radiography, electrocardiogram, and vital signs, were uneventful, except for mild hypertension noted by an internist. In addition, airway management was predicted to be difficult based on the following preoperative airway findings: cutaneous laxity (Figure 1, left) and mandibular micrognathia (Figure 1, right) caused by neurofibromatosis of von Recklinghausen's disease and trismus resulting from pathological fractures associated with mandibular osteomyelitis (Figure 2).

\section{Anesthetic Course}

Considering the airway findings, mask-to-face ventilation after anesthesia induction was deemed inapplicable; therefore, we chose fiberoptic intubation while maintaining spontaneous ventilation under sedation. When the patient entered the operating room, standard monitors were attached. No major problems were noted with her vital signs, except for the systolic blood pressure of between 180 and 190. After establishing an intravenous line in the left forearm and adequate oxygenation at $6 \mathrm{~L} / \mathrm{min}$, we administered $2 \mathrm{mg}$ of midazolam and $50 \mu \mathrm{g}$ of fentanyl. Once sedated, her systolic blood pressure decreased to 120-130. After confirming sufficient sedation and spontaneous breathing, we performed cricothyroid cartilage puncture under ultrasound guidance using $4 \%$ xylocaine. After puncture, awake intubation was performed using a fiberscope from the right nasal cavity. The intubation was successful. Thereafter, anesthesia was induced with $50 \mathrm{mg}$ of propofol and $40 \mathrm{mg}$ of rocuronium. Intraoperatively, anesthesia was maintained using oxygen, air, desflurane, fentanyl, and remifentanil. Postoperatively, the patient recovered from the muscle relaxants, as confirmed by neuromuscular monitoring. Then, $150 \mathrm{mg}$ of sugammadex, a rocuronium antagonist, was administered. After body movement, eye opening, adequate spontaneous breathing, and obedience were confirmed, she was extubated. Subsequently, the systolic blood pressure increased to 180-190. Other vital signs remained within the normal range. The operation lasted for 1 hour and 4 minutes, with 2 hours and 12 minutes of anesthesia. The amount of blood loss was $30 \mathrm{~g}$.

\section{Discussion}

As described above, various problems are associated with anesthesia management in patients with von Recklinghausen's disease. In the present case, the worst problem in anesthesia management was the difficulty in establishing an intact airway. Generally, mask-to-face ventilation is difficult or impossible in patients with upper-airway deformity because of several factors, including tumor, abscess, radiation history, and surgery history ${ }^{9,10}$; thus, awake intubation is often indicated. Although awake intubation is safe because it is performed while maintaining the patient's spontaneous breathing, it must be chosen after careful consideration because it can be painful if the anestesiologist is not skilled. The procedure cannot be performed without patient cooperation. In addition, persistent airway injuries have been reported. ${ }^{11}$ In a previous case report, a patient with airway difficulty was anesthetized using remimazolam, which has become clinically available recently in Japan, but was later awakened because mask-to-face ventilation was impossible. ${ }^{12}$ In our case, 
mask-to-face ventilation was also impossible because of cutaneous laxity caused by neurofibromatosis, which is a characteristic of von Recklinghausen's disease. Therefore, we had to choose awake intubation. The use of midazolam, fentanyl, and a local anesthetic agent to reduce the patient's pain allowed an uneventful awake intubation. As a precautionary measure, a tube is inserted from the nasal cavity. The tube's tip is placed close to the glottis, serving as an airway if oxygenation is reduced due to insufficient spontaneous ventilation during tracheostomy under sedation. Fortunately, such a tube was not needed in the present case. A sedative agent with minimal respiratory depression, such as dexmedetomidine, may be a better choice when the impossibility of mask-to-face ventilation is suspected.

Another problem we encountered during anesthesia management was the increase in systolic blood pressure, which is similar to a previous report. ${ }^{13}$ Previous reports have documented cases of massive bleeding caused by blood vessel fragility, which is a characteristic of von Willebrand disease, in addition to difficulty in intraoperative blood pressure management. ${ }^{13}$ In our case, the systolic blood pressure was elevated to 180-190 owing to tension upon entering the operating room and stimulation during extubation. Thus, the possibility that the patient had hypertension concomitantly cannot be ruled out. The patient's blood pressure was slightly high before the operation, which was noted by an internist. Therefore, preoperative interventions, such as the administration of antihypertensive medication, should have been performed. Although no major circulatory changes were noted intraoperatively, adequate consideration of the circulatory system may be necessary even if the patient did not report any problems.

Furthermore, the effects of muscle relaxants persist in some cases. In our case, the muscle relaxant effect subsided although it was monitored continuously by neuromuscular monitoring during the operation. To ensure safety, we reversed the muscle relaxant before awakening the patient from anesthesia. In the case we experienced, there was no residual muscle relaxant, but since the tracheal intubation was performed under conscious intubation without muscle relaxants, it may not have been necessary to use muscle relaxants intraoperatively and risk their prolongation.

\section{Conclusion}

We experienced the perioperative management of a patient with suspected difficulty in airway management because of cutaneous laxity caused by neurofibromatosis of Recklinghausen's disease and trismus resulting from pathological fractures associated with mandibular osteomyelitis. Although we were able to secure the airway by conscious intubation without any problems, there were some points to be considered regarding intercirculatory management and the use of muscle relaxants.

\section{Acknowledgements}

Not applicable

\section{Funding}

None

\section{Conflict of interest}

The authors declare that we have no conflict of interest.

\section{Data availability statement}

Not applicable due to patient privacy concerns.

\section{Author's contributions}

All authors contributed to the case. Preoperative management and anesthetic planning were performed by AS, IK, YO, MH and NT. Anesthetic Management was performed by AS and IK. The Operation was performed by HI. The first draft of the manuscript was written by AS and all authors commented on previous versions of the manuscript. NN and MO helped with the supervision of the manuscript and development of the overall periopertative plan. All authors read and approved the final manscript. 


\section{Ethical approval}

Not applicable.

\section{Consent for publication}

The authors obtained written consent for publication from the patient.

ORCID Aiji Sato (Boku) 0000-0003-0182-2543

\section{References}

Von Recklinghausen FD. Ueber die multiplen Fibrome der Haut und ihre Beziehung zu den multiple Neuromen. Berlin: Hirschwald; 1882.

Niimura M. Overview and clinical aspects of neurofibromatosis 1. Gann Monograph on Cancer Reserch. 1999; 46: 115-126.

3) Cho T, Fukushige T, Taze C, et al. An experience of anesthesia of Recklinghausen disease leading to massive hemorrhage. J Clin Anes (Japan). 1987; 11:62-64. (Article in Japanese)

4) Steen TR. Hypertension during operation due to unsuspected pheochromocytoma associated with neurofibromatosis. Anaesthesia. 1957; 2: 80.

5) Nishiyama T, Odaka Y, Akao M, et al. Experience in anesthesia of mediastinal tumor excision in a patient with Recklinghausen disease. J Clin Anes (Japan). 1990; 14: 427-428. (Article in Japanese)

6) Tominaga S, Shiraishi T, Akiyama Y, et al. A case of general anesthesia for a patient with von Recklinghausen syndrome and tumors of the oral cavity. J Jpn Dent Soc Anesthesiol. 1988; 16: 305-308. (Article in Japanese)

7) Magbagbeola JA. Abnormal responses to muscle relaxants in a patient with Von Recklinghausen's disease (multiple neurofibromatosis). Br J Anaesth. 1970; 42: 710.

8) Manser J. Abnormal responses in von Recklinghausen's disease. Br J Anaesth. 1970;

42: 183.

9) Kheterpal S, Martin L, Shanks AM, et al. Prediction and outcomes of impossible mask ventilation: a review of 50,000 anesthetics. Anesthesiology. 2009; 110: 891-897.

El-Orbany M, Woehlck HJ. Difficult mask ventilation. Aesth Analg, 2009; 109: 1870-1880.

Maktabi MA, Hoffman H, Funk G, et al. Laryngeal trauma during awake

fiberoptic intubation. Anesth Analg. 2002; 95: 1112-1114.

Sato A, Hashimoto M, Tachi N, et al. Experience with remimazolam in two cases in which difficult airway management was predicted. J Clin Anes (Japan). 2021; 45: 1346-1348. (Article in Japanese)

Tanigawa H, Oomizo H, Naito M, et al. Anesthesia experience in a case of von Reclinghausen's deisease in when the operation had to be concluded prematurely. J Jpn Dent Soc Anesthesiol. 1997; 25: 251-255. (Article in Japanese)

\section{Figure Legends}

Figure1

Cutaneous laxity (left) and mandibular micrognathia (right) caused by neurofibromatosis of von Recklinghausen's disease

Figure2

Pathological fractures associated with mandibular osteomyelitis 

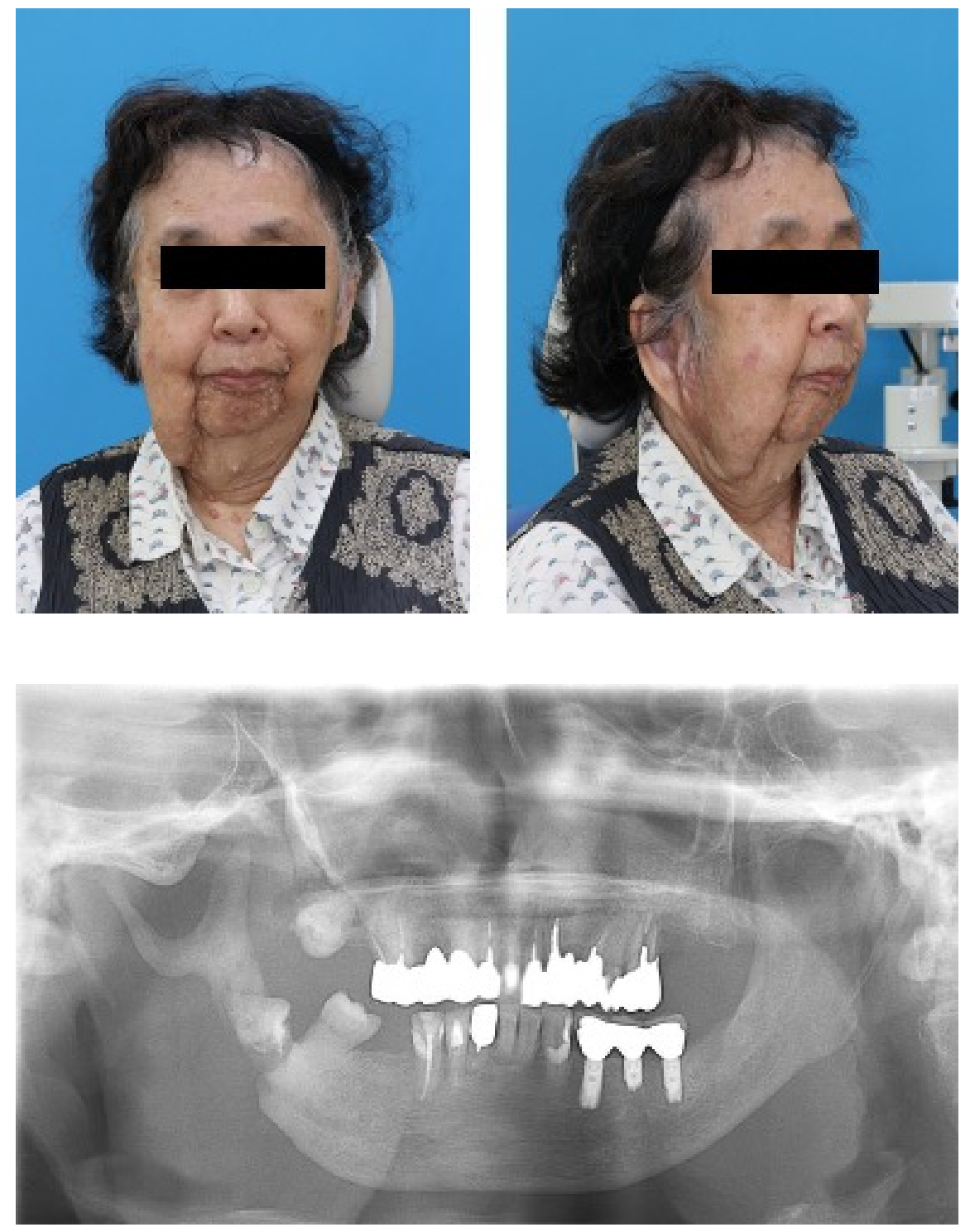